\title{
Cooperative Communication for Wireless Sensors Network : A Mac Protocol solution
}

\author{
Bastien Mainaud, Vincent Gauthier and Hossam Afifi \\ Wireless Networks and Multimedia Services Department \\ Institut Telecom Sud-Paris \\ 9, rue Charles Fourier \\ 91011 Evry cedex, FRANCE \\ Email: \{bastien.mainaud,vincent.gauthier,hossam.afifi\}@it-sudparis.eu
}

\begin{abstract}
This article proposes a new Mac layer scheme (WSC-MAC) for Wireless Sensor Network (WSN) improving the overall the network reliability by using cooperative communication. We focus our work on a way to define a relay node among the neighborhood of a node, efficiently and with only few signaling messages. We developed a solution based on an automatic forwarder selection and a link state evaluation in order to define the relay node. This automatic selection uses a group identifier uniformly spread in the network and ensures that only few nodes at the time will be chosen as relay. As the sensor nodes switch from active mode to sleep mode, we based our solution on the Long Preamble Emulation (LPE) Mac layer Algorithm which emulates the asynchronous MAC protocol proposed in [13]. Our Simulations results show that WSC-MAC increases the overall reliability of the sensor network and adjusts to large variety of node density.
\end{abstract}

Index Terms - Wireless Sensor Network, MAC Protocol, Cooperative communications, Reliability

\section{INTRODUCTION}

The motivation of this paper is to fill the gap between cooperative communications technique developed for physical layer and an appropriate MAC layer scheme for Wireless Sensor Networks (WSN). The cooperative radio techniques, also known as Virtual Multiple Input Single Output (VMISO)[9], originate from works using diversity techniques with colocated multiple antennas. Instead of using multiple antennas to take advantage of the diversity, the cooperative communication uses multiple nodes equipped with a single antenna using distributed coding scheme to achieve similar gains. The cooperative communication scheme is also inherently a network solution, and there are issues at multiple levels of the network stack to solve in order to reach the gain offered by the diversity. This paper mainly focus on finding the good tradeoff between the issues at the Mac layer and the physical layer performances. We developed a Mac layer scheme that used a distributed algorithm to select a relay node in an efficient way without extra overhead in signaling and processing. The reliability of the communications that use cooperation helps us to design an acknowledgment agnostic solution. The paper is organized as follows. In section 2, we present the related work. In section 3 , we introduce the design of our solution and the specific mechanisms we developed. In section 4, we present performance results and an analysis. Finally we conclude the paper and present some future works.

\section{RELATED WORK}

Recently, a new class of radio diversity techniques called cooperative communication derived from diversity techniques using co-located antennas has received lot of interests. Laneman et al. [9] and others [2,6] have developed a set of cooperative communication scheme for distributed wireless network like: ad hoc networks or sensors networks. Theirs respective works has paved the way for a lot of studies using cooperative transmission on a real Mac layer framework. Ji et al. [8] and Lin et al. [10] proposed different framework for Cooperative MAC protocol. These solutions are based on network-assisted diversity multiple access (NDMA). These Authors present a novel throughput-efficient medium access scheme for WSN. This scheme enables a node to retrieve a packet from many previously received packets (MPR [15]).

In [11] Liu et al. proposed the first cooperative MAC protocol called "CoopMac" based on the well knows IEEE 802.11 protocol. They defined two alternative solutions CoopMAC I and CoopMAC II. In CoopMAC I, a new frame HTS (Helper ready To Send) is added to the IEEE 802.11, to inform others that an alternative node (a relay node) will help the sender to transmit more efficiently. Then, in CoopMAC II, HTS frame is not used; instead they used the RTS header to advise which node should act as a relay node.

Chou et al. [5] present a solution to perform cooperative communication in distributed wireless networks. Authors claim that only one relay must participate in the cooperative transmission. In order to select the relay node among its neighbors, they developed mechanisms such as a busy tone and a special RTS (Relay-RTS). This RRTS is used with the classic RTS/CTS mechanism to inform the source and the relay node chosen.

Most of these solutions used extra messages in order to setup the cooperative process and select the relay node. In a WSN context, where the resources are limited, the use of these signaling packets should be avoided to reduce the powerconsumption.

\section{Cooperative Communication Design}

Cooperative communications is a promising technique that would enhance the design of the wireless sensor networks. In this context, we have to face also new challenges that need to 
be solved in order to design a full functional system. One of these challenges is to find a way to select a relay node that will efficiently forward packets in a cooperative way, in order to reach an improvement on channel capacity. In the following section of this document, we present our solution based on an automatic forwarder selection and a Link-state evaluation that enable our MAC layer to define some neighbors as relay node.

\section{A. Automatic forwarder selection}

Nodes in cooperative network are efficiently using broadcast property of the wireless medium to improve the communication between two nodes in the networks by sending on the same channel a copy of the main communication. To reach the trade off between gain brought by the cooperation and the overhead introduced, Fan et al. [7] and Moh et al.[12] show that the best cooperation happens in the case of a few nodes cooperation instead of a multiple-nodes cooperation pattern. Based on this observation we have developed an algorithm that enables the node that want to send a packet to pre-define a small set of relay nodes that will help to forward packets to the destination.

To select some possible relay nodes our algorithm introduces a new ID: a Group Identifier, which is an ID embedded in the sensor (different from the node address; and not unique in the network). The Group Identifier is enabling during the auto-configuration process by each WSN. Each time a node want to send a packet, it should first draw a random number among the possible value of Group Identifier, put the result inside the packet header and sends it to the destination. After, all the nodes hearing the packet be broadcasted on the channel have to check the group identifier included into the packet header. If the identifier into the packet header matches with their own predetermined Group Id, they could become a relay for this communication. Otherwise, the packet will be dropped. As example in figure coop2, the group identifier draw by the node $\mathrm{S}$ is 1 and the node $\mathrm{R}$ and $\mathrm{T}$ which are in that group should now be considered to forwarding the packet.

To set up its own Group Identifier each WSN node picks a random number uniformly distributed between 0 and $\mathrm{A}$, the average number of neighbors in the network calculated as a function of the network size $\mathrm{R}$ and the number of nodes present in the networks. We are using group identifier to limit the number of relay nodes of each communication. We assume that the number of relay nodes will be on average close to one, the optimum number of relay. This identifier will be set up during the network deployment and will be tie to the network topology. In order to avoid any collision, two nodes in the same neighborhood should not have the same Group ID (a node may have two neighbors with the same group ID), the auto-configuration process should take into consideration the group ID of its neighbors when it determines its own group ID (cf. Algorithm 1). Algorithm 1 presents the process in order to draw the group identifier of each node. In our algorithm, A_list is a list of $A$ available values for group identifier.
Input: A : average number of neighbors

Output: my_group_ID

create $A \_l i s t$ using $A$; my_group_ID $=0$;

while (my_group_ID $=0$ ) do

listen the channel for a random period;

if (a neighbor send a Group_ID) then

I I should take off the Group Identifier of A_list; A_list $=$ A_list - Group_ID;

else

I pick a random value among $A_{-}$list for

my_group_ID;

if $\left(A \_\right.$list $\left.=N U L L\right)$ then

| my_group_ID = random(A_list);

else

create $A \_l i s t$ using $A$;

end

broadcast(my_group_ID);

end

end

Algorithm 1: Algorithm process on each node for drawing its own group identifier

\section{B. Link-State Evaluation}

In the previous section, we established a distributed strategy to pick a possible relay node among the neighborhood of another node willing to send a packet. But in order to be efficient, the cooperation should occur if and only if the cooperative communication enhances performance of the transmission as well for the overall performance. So to know if the cooperative communication, with the help of a relay node, would improve the communication, we design a link state algorithm. The Link State algorithm is running on each node that has been elected to act as a relay node during automatic forwarding selection process. This process will help the elected relay to determine if it should be involved into the relaying process. The relay node will estimate the channel quality of its link

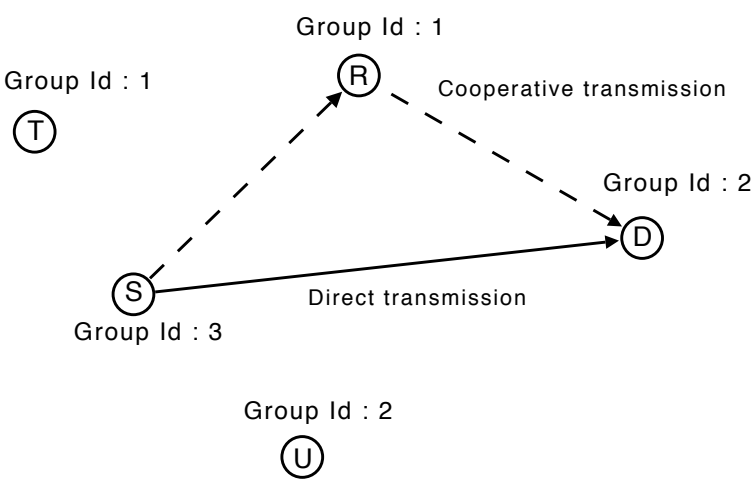

Fig. 1. Cooperative communication scenario 
towards the destination and will compare it to the link quality from the source to the destination. For that, each node stores a Link_State table, this table contains the quality of each link it has with every neighbor. If the link quality is better than the link quality to the source, we use the cooperation. To estimate and process the channel quality the elected nodes will retrieve the RSSI (Receive Signal Strength Indicator) from the physical layer. The RSSI measured by the 802.15.4 devices enable the algorithm to get the link quality indication (LQI) of the channel.

As example in figure 1,the node $R$ and $T$, after their election by $S$ (by checking the Group Identifier embedded in the packet) should consider forwarding the packet from $S$. Node $R$ checks the LQI of its link to $D$ in its Link_State table and computes the capacity of this link based on average SNR (Signal Noise Interferences Ratio), and decides if it will forward the packet from $S$. Our solution does not provide any guaranties on the fact that only one node at the time would act as relay per communication, but the number of relay per communication is close to one if the automatic forwarder process is well configured.

To design our link state evaluation algorithm and the LQI, we essentially based our work on both the characteristics of the wireless sensor network physical layer used in the IEEE 802.15.4 standard [1] to retrieve the bit error rate and Space-Time-Coded Cooperative Diversity scheme defined by Laneman et al. [9] Assuming these two bases, the relay node is able to characterize the transmission quality between twoneighbor nodes with and without its cooperation. Considering the Maximum Ratio Combining Diversity [3] the SNR of the diversity combiner is the sum of the SNR of each transmission. Then, we can determine the bit error rate of a cooperative transmission. For the capacity of the channel in cooperative transmission, we sum the mutual information and obtain equation 1 where $\mathrm{W}$ is the bandwidth as defined in IEEE 802.15.4 [1] SNR_rd is the SNR between the relay and the destination and SNR_sd is the SNR between the source and the destination. Derived from the formula 1 and the information stored by the relay node (LQI), the relay is now able to take the decision of forwarding the packet. The forwarding process could be triggered by a couple of criteria like the capacity of the channel or the transmission error rate. Algorithm 2 summarizes the processes that occur during the reception of a packet by a random node into the network.

$$
\begin{array}{r}
\operatorname{Capa}_{\text {coop }}=W * \frac{1}{2}\left[\log \left(1+S N R_{r d}\right)+\right. \\
\left.\log \left(1+S N R_{s d}\right)\right]
\end{array}
$$

C. MAC layer protocol details for WSN using cooperative communication

We based our solution on a CSMA-like MAC Layer adapted to the WSN [13], where the duty cycle has been reduced as much as possible to lower the overall energy consumption. To reduce consumption from idle-listening a well known technique consists of using a preamble in order to inform the other nodes that a packet will be sent [4,13]. For our purpose to initiate the cooperative communication the sleep phase could be a problem, because some of neighbors could be involved in the relaying process. Nodes who hear a preamble should process the algorithms previously described with the intention of participating to the cooperative process. In Figure 2 , we present a frame sending sequence of our protocol. Node $S$ used a preamble for the synchronization with its neighbors then, the cooperative communications occurs in two steps. : the first is the transmission of the packet from the source to the destination, then all nodes hearing the message should check if they have to consider forwarding it. In the second step, using the Space-Time-Coded Cooperative Diversity, the packet must be sent by the source and the relay at the same time.

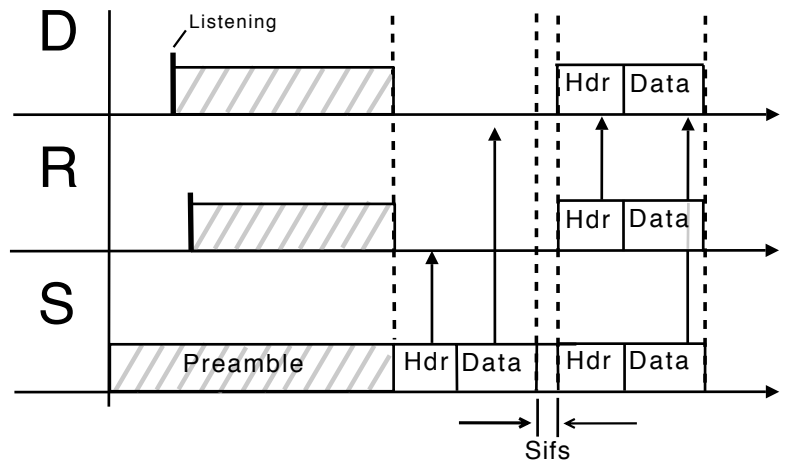

Fig. 2. Frame sending sequence between a source $S$, a relay $R$ and a destination D

\section{PERFormance ReSUlts AND ANALYSis}

This subsection presents simulation results showing the behavior of a simulated wireless sensor network taking advantage of cooperative communications. To quantify the performance of our proposed cooperative communication scheme, we have developed a network simulator with Matlab [14].

Our simulator implements cooperative communication model previously defined in [cf. Lanman et al [9]] in addition to our proposed algorithm (cf. Algorithm 2). Each plot point presented here is an average calculated with all data coming from all the possible communication into a network of 100 nodes over 450 runs (30 simulation for 15 different topologies) with a $95 \%$ confidence interval. For the sake of clarity we have summarized the simulations settings in Table I.

Packet delivery ratio: We decided to highlight different aspect of our proposed solution as function of the network density, because the performance of our Mac layer proposal will rely on the probability that a cooperative communication could be found to improve the overall performance. The probability that a node could be used as relay is closely tied to the network density. In figure 3 we show the PDR (Packet Delivery Ratio) as function of the network density and we could see an improvement of an order of $10 \%$ where our network is not dense. Meaning that in the case of a high density network our solution does not give any improvement, 


\section{Input: Packet PktI \\ Output: Packet PktI}

receiving a packet;

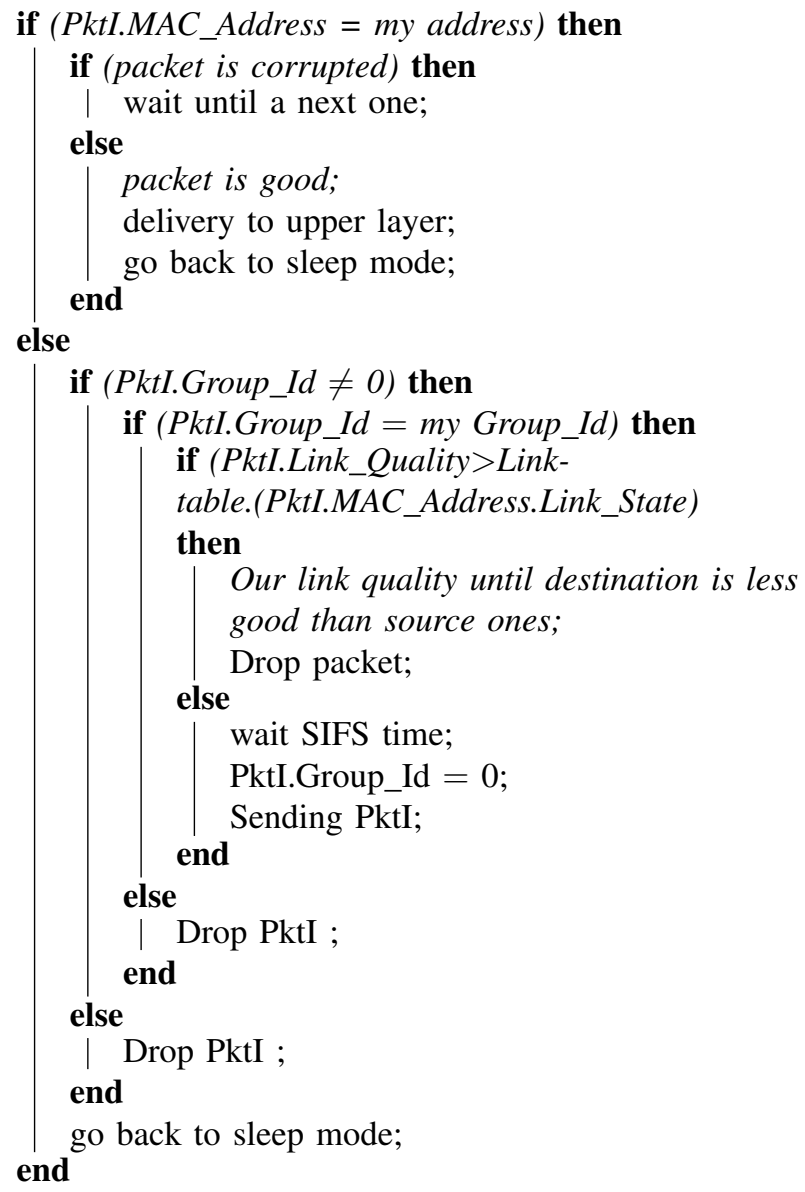

Algorithm 2: Algorithm process on each neighbor receiving a packet

TABLE I

SUMMARY OF SimULATION PARAMETERS

\begin{tabular}{ll}
\hline Parameter & Value \\
\hline Simulations Parameters & \\
Number of nodes & 100 \\
Nodes density (nodes $/ \mathrm{m}^{2}$ ) & $0,01-0,25$ \\
number of topologies & 15 \\
number of iterations & 30 \\
MAC/PHY Parameters & \\
Typical Transmission Range (meters) & 35 \\
Attenuation factor & 3 \\
Pathloss Model & Free Space \\
Physical Model & 802.15 .4 \\
Receiver Sensitivity (dbm) & -90 \\
Packet size (bits) & 200 \\
Acknowledgment size (ACK/NACK) (bits) & 40
\end{tabular}

because of the density there is always a links to deliver packets with a high probability of success due to the close distance between nodes.

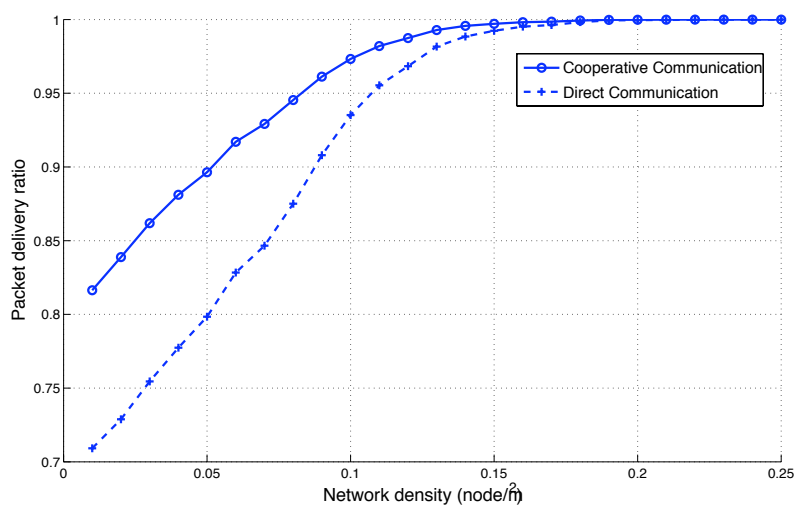

Fig. 3. Packet delivery ratio as function of the nodes density

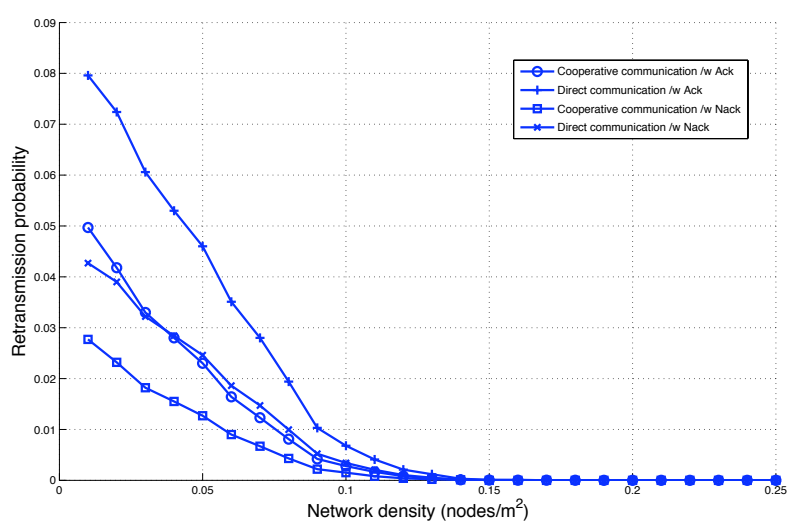

Fig. 4. Retransmissions probability (with ACK and NACK) as function of the nodes density

Reliability: In figure 4, we are showing a comparison between two different retransmissions schemes using the cooperative communication: ACK and NACK. As result, we could see that the NACK scheme in the case we are using cooperative communication outperform the others by an order of magnitude of $66 \%$ and $55 \%$ in the two different case of figure.

Network capacity: Figure 5 shows us the average channel capacity as function of the density. We can see that the capacity reaches its maximum when the network density is close to 0.08 node ${ }^{2}$. Note that the cooperative capacity is far beyond the real sensor nodes capacity but this gives a information about the possible performances of our solution [6].

Figure 6 shows the number of packet exchanged by nodes into the network, including the retransmissions for three different nodes behaviors: all the nodes used direct communication only; the nodes used our proposed communication pattern and algorithms; and in the last case, we plot the number 


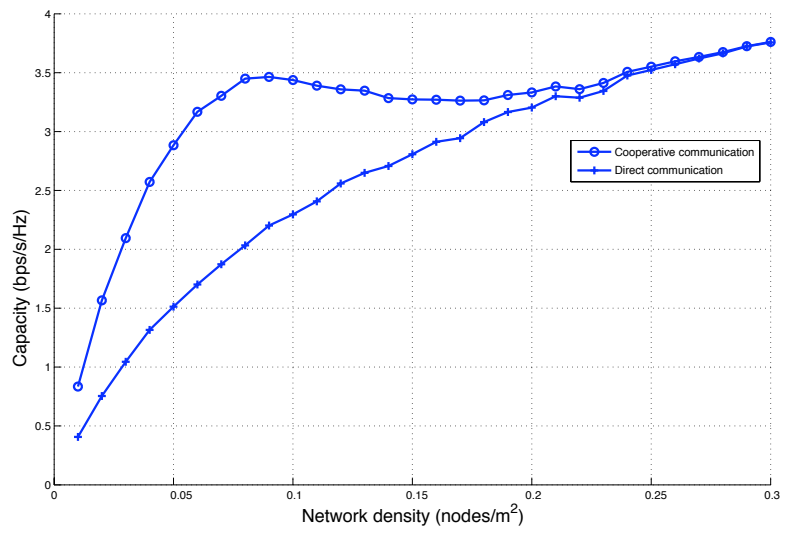

Fig. 5. Capacity as function of the nodes density

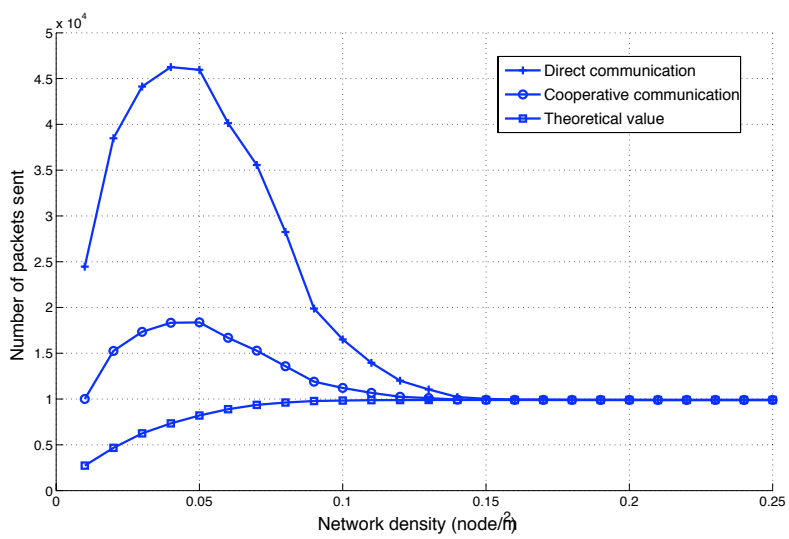

Fig. 6. Comparison of the number of packets sent and the theoretical value as function of the nodes density

of transmission in case of a perfect environment (direct communication with no loss, no retransmission, etc). In a low density topology, the direct communications use a lot of bandwidth. There are a lot of packets lost and so there are a lot of retransmissions. Using cooperative communications, the number of packets sent is lower, and so, it allows to save some bandwidth.

Our simulations show that WSC-MAC enhances the packet delivery ratio and the reliability of the network in the case of a low dense network(lower than 0.10 nodes $/ \mathrm{m}^{2}$ ). The acknowledgment traffic is also reduced by our solution. The capacity using cooperative communication is far beyond the real sensor nodes capacity pointing out that some research need to be done in adaptive modulation dedicated to sensors networks.

\section{CONClusion AND Future WORK}

In this paper, a new cooperative MAC protocol tailored for WSN is proposed. In order to fulfill the set of constraints imposed by the cooperative communication scheme and the wireless sensors scheme we have developed an algorithm allowing the automatic selection of the forwarder node (relay node) using only few message exchanges during the network setup phase. To optimize the selection of the relay node algorithm we are using a cross-layer design to fetch information from the physical layer. Our simulations show that the proposed solutions brought enhancements (packet delivery ratio) and reliability to the network in the case of a low dense network. Nevertheless in case of a massively dense network, the use of cooperation techniques do not bring any enhancements and even will have a negative impact on the performances. This is due to the fact that most of wireless links in the network are good enough to carry traffic with very few loss provoked by interferences coming from others transmitting node. This issue is not balancing the overload of a cooperative communication. This concern lead us toward fact that any Mac layer scheme that are exploiting cooperative communication should be used in adaptive way, in order to be efficient in any case. In our future works we will focus on optimizing the group identifier decision process with the aim of finding an even more efficient selection of a relay nodes also well suited for sensor networks.

\section{REFERENCES}

[1] IEEE std 802.15.4-2006 (revision of ieee std 802.15.4-2003). 2006.

[2] S.M. Alamouti. A simple transmit diversity technique for wireless communications. IEEE J. Sel. Areas in Comm., 16(8):1451-1458, Oct 1998.

[3] D.G. Brennan. Linear diversity combining techniques. In Proc. of the Institute of Radio Engineers, 47(6):1075-1102, June 1959.

[4] M. Buettner, G. V. Yee, E. Anderson, and R. Han. X-mac: a short preamble mac protocol for duty-cycled wireless sensor networks. In Proc. of the 4th international conference on Embedded networked sensor systems (SenSys '06), pages 307-320, New York, NY, USA, 2006.

[5] C.T. Chou, J. Yang, and D. Wang. Cooperative mac protocol with automatic relay selection in distributed wireless networks. In Proc. of the Fifth IEEE International Conference on Pervasive Computing and Communications Workshops (PERCOMW '07), pages 526-531, 2007.

[6] M. Dohler, A. Gkelias, and A.H. Aghvami. Capacity of distributed phylayer sensor networks. IEEE Trans. on Vehicular Technology, 55(2):622639, March 2006

[7] Y. Fan and J. Thompson. Mimo configurations for relay channels: Theory and practice. IEEE Trans. on Wireless Comm., 6(5):1774-1786, May 2007.

[8] W. Ji and B. Y. Zheng. A novel cooperative MAC protocol for WSN based on NDMA. In Proc. of The 8th International Conference on Signal Processing (ICSP'06), pages 16-20, 2006.

[9] J. N. Laneman and G. W. Wornell. Distributed space-time-coded protocols for exploiting cooperative diversity in wireless networks. IEEE Trans. on Inf. Theory, 49(10):2415-2425, 2003.

[10] R. Lin and A.P. Petropulu. A new wireless network medium access protocol based on cooperation. IEEE Trans. on Signal Processing, 53(12):4675-4684, Dec. 2005.

[11] P. Liu, Z. Tao, and S. Panwar. A cooperative MAC protocol for wireless local area networks. In Proc. of the International Conference on Communications (ICC 2005), volume 5, pages 2962-2968, 2005.

[12] S. Moh, C. Yu, S.-M. Park, H.-N. Kim, and J. Park. Cd-mac: Cooperative diversity mac for robust communication in wireless ad hoc networks. In Proc. of the International Conference on Communications (ICC '07), pages 3636-3641, June 2007.

[13] J. Polastre, J. Hill, and D. Culler. Versatile low power media access for wireless sensor networks. In Proc. of the 2nd international conference on Embedded networked sensor systems (SenSys '04), pages 95-107, 2004.

[14] MATLAB R2007a. http://www.mathworks.com, 2007.

[15] L. Tong, Q. Zhao, and G. Mergen. Multipacket reception in random access wireless networks: From signal processing to optimal medium access control. IEEE Communication magazine, 39(11):108-112, Nov 2001. 